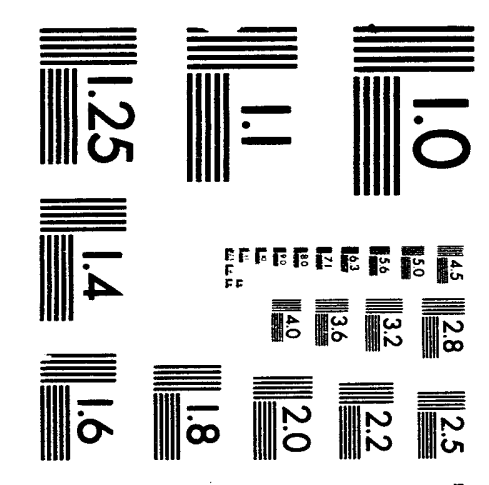



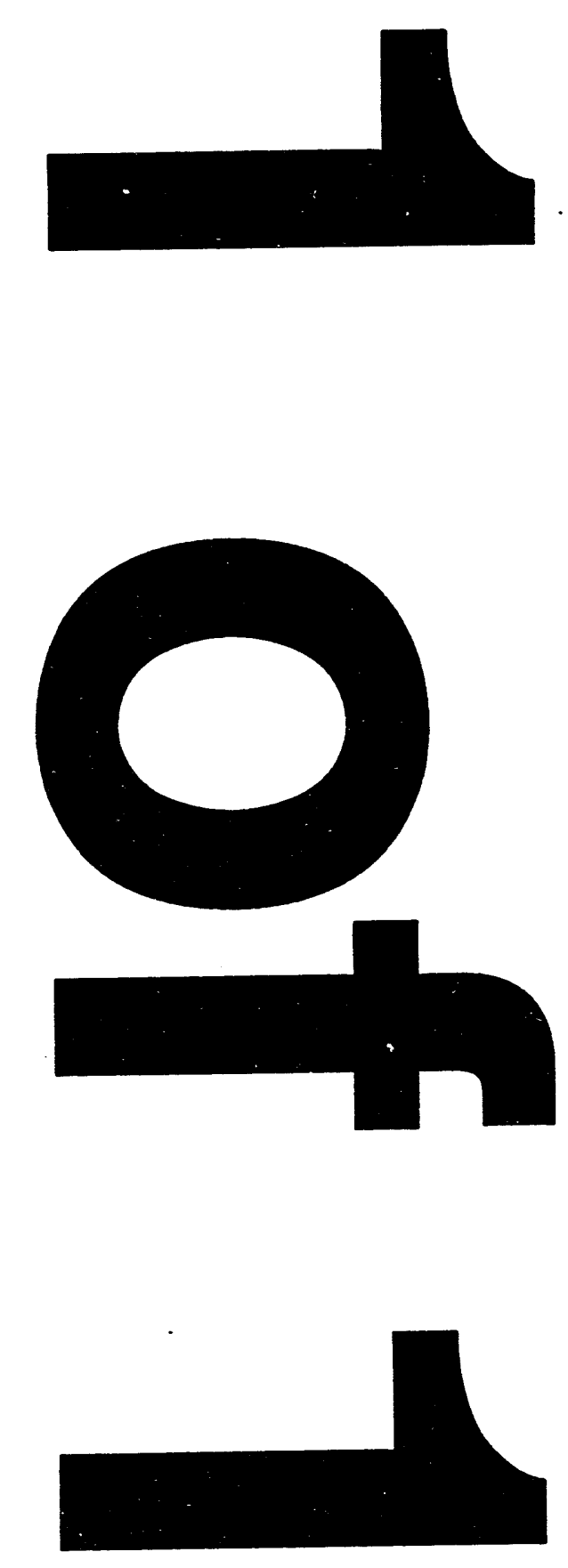


\section{ASYMPTOTIC COHERENCE OF GLUONS AND OF q-BOSONS*}

\author{
Charles A. Nelson
Department of Physics \\ State University of New York at Binghamton \\ Binghamton, N.Y. $13902-6000$
}

\section{ABSTRACT}

In theoretical physics one of the most important aspects of coherent states is that they can often be simply and reliably used to investigate the quantum coherence and correlation properties of new dynamical, quantum field theories. First, this paper reviews the coherent/degenerate state treatment of the infra-red dynamics of perturbative QCD. This is based on the asymptotic behavior of the QcD. This is based on the asymptotic behavior of the Hamillonian operaror as $\mathrm{IH} \rightarrow \infty$ in the interaction representation. Second, the paper reviews the usage of q-analogue coherent states $|z\rangle_{q}$ to deduce coherence and uncertainty properties of the $q$-analogue quantized radiation field in the $|z\rangle_{q}$ "classical limit" where $|z|$ is large. Third, for future applications, a new "projector" definition of the usual coherent states and of the squeezed states is reported.

\section{Introduction}

This paper is written so that a reader can focus on whichever of the three on-going CS research topics she/he is interested in. Here we provide what are essentially "extended abstracts" for each. To balance our discussions, we strongly urge the reader to examine the listed references. Particularly in the case of the first topic, there is a very extensive literature in QCD and in QED.

(i) As in QED, QCD pertubation theory is plagued by infrared divergences. ${ }^{1}$ In both theories, coherent infrared-finite asymptotic states and associated S-matrix amplitudes can be systematically constructed. ${ }^{2-7}$ On the other hand, at the "cross-section level" in most QCD calculations, singularities due to soft (low) momentum gluons do indeed already cancel by the Bloch-Nordsieck mechanism, 1,8 much as in $Q E D$, when the fina state is sufficiently inclusively defined. Therefore, neglecting $\mathrm{O}\left(\mathrm{m}^{2} / \mathrm{Q}^{2}\right)$ corrections, it has been possible to generalize the parton model ${ }^{9}$ in QCD to a factorizable formalism ${ }^{10} \mathrm{n}$ for hadron-hadron scattering, in which the partons are identified as the quarks and gluons. However, counter-examples to this BN-cancellation were discovered in 1980 by Doria, Frenkel, and Taylor ${ }^{12}$ at the two-loop level at higher twist, i.e. when $\mathrm{O}\left(\mathrm{m}^{2} / \mathrm{Q}^{2}\right)$ corrections are included where $\mathrm{m}^{2}$ is the quark mass scale and $\mathrm{Q}^{2}$ the square of the process's hard four momentum. These counter-examples 11,12 include quark-antiquark annihilation into an off-mass-shell photon, and inclusive quark-quark scattering. These counter-examples prompt questions concerning the validity of arguments for the factorization and universality of mass singularities in hard scattering processes because the arguments assume that no length cutoff, e.g. associated with the spatial extent of a hadron, is necessary to insure cancellation of IR divergences.

\section{CNELSON@BINGVMB}

The substance of this review is not new, but the material may be of pedagogical use to some readers.
The non-cancelling divergence, however, can be removed by including initial soft - gluons with the initial quarks using either initial (energy) degenerate states, ${ }^{14,13,15}$ or it - can be avoided by using the above-mentioned, initial quark-gulon coherent states. ${ }^{16}$ In the CS case, it is sufficient to dress each quark leg separately so that there are parton "jets" in the initial state. ${ }^{17}$ It is not necessary for there to be a residual, asymptotic interaction connecting the initial quark and antiquark legs. By this construction, we obtain an asymptotic Fock space of colored, coherent parton "jets."

Therefore, it was proposed ${ }^{18}$ that the partons be identified with nearly degenerate, coherent quark-gluon "jet" states. For instance, in deep-inelastic lepton-hadron scattering, incoming quark states are replaced by soft and collinear degenerate jet states and the Bjorken $x$ variable is reinterpreted to be the momentum fraction of a "jet" in the initial hadron. Explicit caiculation ${ }^{18}$ shows that such a formalism is possible in QCD in the framework of the operator product expansion.

(ii) In 1989, q-analogue coherent states, $\mid z>$, were introduced in association with the construction of the q-oscillator realization 19,20 of the remarkable symmetries of the new "quantum algebras." The q-analogue CS's are eigenstates of the q-boson's annihilation operator, $a|z\rangle_{q}=z|a\rangle_{q}$, just as occurs in quantum-optics for the quantized electromagnetic field. It is not yet known whether nature makes use of $\mathrm{q}$-bosons as some sort of non-linear quasi-particle excitation of the ordinary electromagnetic field (or other known fieid) or as the quanta for a brand-liew type of cooperative phenomena. Possibly, the occurrence of q-bosons requires a background lattice or other "material media" for the occurrence of q-bosons requires a background lattice or other "material media" for
their realization as in the case of phomons, etc. In any case, to recognize the presence of such an underlying quantized q-boson field, we need to know the field's coherence properties.

The q-analogue CS's do still minimize the $\mathrm{q}$ and $\mathrm{p}$ uncertainty relation and the $n$-th order field correlation function still factorizes. Using the idea of q-integration, a resolution of unity 21,22 for the q-analogue CS's can be obtained. However, in the $|z\rangle$ resolution of unity ${ }^{21,22}$ for the q-analogue CS's can be obtained. However, in the $|\mathrm{z}\rangle_{\mathrm{q}}$
"classical limit" in which $|z|$ is large, most uncertainty fractions are no-longer $\ll<1$, but are instead of $\mathrm{O}(1)$. For the number operator and free q-boson gas Hamiltonian, the uncertainty fractions do go to zero. Consequently, quantum effects are generally expected to be pervasive in the CS-classical limit, unlike for the usual CS's with $q=1$. Nevertheless, the conventional number-phase uncertainty relation, $\Delta N \Delta \hat{\phi}_{q}=1 / 2$, and the approximate commutation relation $\left[N, \hat{\phi}_{q}\right]=i$ of Dirac are found ${ }^{20}$ to hold in the CS basis $|z\rangle_{q}$ for large $|z|$. Thus, $\mathrm{N}$ and $\phi_{\mathrm{q}}$ are almost canonically conjugate in this limit.

(iii) It is well known that usual CS can be defined in either of three ways: as eigenstates of the bose annihilation operator "a", by acting on the vacuum $10>$ state with $D(z)=\exp \left(\mathrm{za}^{*}-\mathrm{z}^{*} \mathrm{a}\right)$, or by minimizing the $\mathrm{q}$ and $\mathrm{p}$ uncertainty relation. However, CS's can also be defined ${ }^{23}$ using a "projector" operator

\section{MACTFR}

DISTRIBUTION OF THIS DOCUMENT IS UNLIMITED $f 7 \alpha$ 


$$
P=\sum_{n} \frac{(-1)^{n} b^{t_{n}} b^{n}}{n !}
$$

to transform the old vacuum state $10>=10>_{a}$ into a new vacuum state $10>_{b}$, where $b 10>_{b}=$ 0 with $b=a-z$. Explicitly, $P\left|0>_{a}=10>_{b}=e^{-|z|^{2}} e^{z a t}\right| 0>$. Similarly the usual "squeezed vacuum state" $S(\alpha)|0\rangle$, and the usual "coherent squeezed state" $|z, \alpha\rangle \equiv$ $D(z) S(\alpha) 10>$ can be defined using projectors.

\section{Coherent State Approach to Pertubative QCD}

Is the Block-Nordsieck conjecture correct in pertubative QCD with massive quarks? This cross section conjecture is: "If both final real soft gluons and virtual soft gluons are summed, the soft IR (infrared) divergences will cancel in the color-averaged cross section?" This appeared quite reasonable because the analogous conjecture ${ }^{1}$ is true in QED and it's only the pertubative dynamics of "soft" gluons that is being considered for both the reals and the virtuals. However, at $\alpha_{s}^{2}$ order in " $q+\bar{q} \rightarrow \gamma^{*}+$ soft gluons" where $\gamma^{*}$ is a hard photon, a counter-example ${ }^{12,13,24}$ occurs: One obtains

$$
\begin{aligned}
\sigma(\mathrm{q} \overline{\mathrm{q}} & \left.\rightarrow \gamma^{*}+\text { gluons }\right) \underset{\substack{\text { color, and } \\
\text { spin averaged }}}{\mid}=\sigma_{\mathrm{B}}\left\{1+\alpha_{\mathrm{s}}^{2}\left[\mathrm{AC} \mathrm{C}_{\mathrm{F}}+\mathrm{NC}_{\mathrm{F}} \mathrm{C}_{\mathrm{YM}}\right]\right\} \\
& =\sigma_{\mathrm{B}}\left\{1+\alpha_{\mathrm{s}}{ }^{2} \mathrm{C}_{\mathrm{F}} \mathrm{C}_{\mathrm{YM}}(-1 / 4 \epsilon)[(1-\beta) / \beta][1 / \beta \cos ((1+\beta) /(1-\beta))-2]\right\}
\end{aligned}
$$

using dimensional regularization, $\epsilon=(4-n) / 2$, with $\vec{\beta}=\vec{q} / q_{0}$ the velocity of the quark in the antiquark rest frame. Note that the uncancelled divergence as $\epsilon \rightarrow 0$ is in the coefficient of the non-Abelian $C_{F} C_{Y M}$ term. [For $T_{2}=$ quark color matrix, $T_{2} T_{2}=C_{F}$ \} and $\mathrm{f}_{\mathrm{abc}} \mathrm{f}_{\mathrm{abd}}=\mathrm{C}_{\mathrm{YM}} \delta_{\mathrm{cd}}$.\} In the QED limit, $\mathrm{f}_{\mathrm{abc}} \rightarrow 0$ and $\mathrm{C}_{\mathrm{YM}}=0$ so there is no problem in the Abelian case.

The systematic construction $36,37,24,25,5$ of the non-Abelian CS's in QCD proceeds as follows: First, in the interaction representation we obtain an asymptotic Hamiltonian

$$
\begin{aligned}
H_{a s}(t) & =\lim _{t u \rightarrow \infty}\left\{H_{o}+H_{I}(t)\right\} \\
& =H_{o}+V_{a s}(t)
\end{aligned}
$$

$$
\mathrm{H}=\mathrm{H}_{\mathrm{as}}+\mathrm{H}_{\mathrm{I}}^{\prime}
$$

such that $\mathrm{H}_{\mathrm{I}}^{\prime}$ vanishes sufficiently fast asymptotically to avoid IR divergences. Since the quark mass is finite, $H_{2 s}(t)$ does not contain $q \bar{q}$ creation or annihilation terms. It does include the complete cubic and quartic gluon self-coupling terms. Second, we obtain the associated time-evolution operator in the Schrödinger representation

$$
\begin{aligned}
& U_{a s}(t)=\exp \left(-i H_{0} t\right) Z(t) \\
& i d / d t Z(t)=V_{a s}(t) Z(t)
\end{aligned}
$$

The pertubative solution can be written as a CS operator exponentiation (Magnus' theorem)

$$
Z(t)=\exp [\Omega(t)], \Omega(t)^{\nmid}=-\Omega(t)
$$

$$
\begin{aligned}
\Omega(t) & =-i \int t \mathrm{~V}(\tau) \mathrm{d} \tau-1 / 2 \int \mathrm{t} d \tau \int^{\tau} \mathrm{d} \sigma[\mathrm{V}(\tau), \mathrm{V}(\sigma)] \\
& +(-i)^{3} 1 / 4 \int \mathrm{d} d \tau \int^{\tau} \mathrm{d} \sigma \int \sigma \mathrm{d} \rho[\mathrm{V}(\tau),[\mathrm{V}(\sigma), \mathrm{V}(\rho)]] \\
& \left.+(-i)^{3} 1 / 12 \int \mathrm{t} d \tau \int^{\tau} \mathrm{d} \sigma \int^{\tau} \mathrm{d} \rho[\mathrm{V}(\tau), \mathrm{V}(\sigma)], \mathrm{V}(\rho)\right]+\ldots
\end{aligned}
$$

Equivalently, in time-ordered pertubation theory

$$
Z(t)=1+\sum_{n=1}^{\infty}(-i)^{n} \int t d_{1} \int t_{t} d t_{2} \ldots \int t^{n-1} d t_{n} V_{a s}\left(t_{1}\right) \ldots V_{a s}\left(t_{n}\right)
$$

Third, the asymptotic states space is constructed by $\left(\left|\Psi_{F}\right\rangle\right.$ is in the interaction representation)

$$
\left|\Psi_{\mathrm{as}}\right\rangle=\lim _{t_{2} \rightarrow-\infty} Z^{\chi}\left(t_{2}\right)\left|\Psi_{\mathrm{F}}\right\rangle
$$

and the S-operator by

so as to rewrite the total Hamiltonian as 


$$
S^{g}\left(t_{1}, t_{2}\right)=\lim _{\substack{t_{1} \rightarrow \infty \\ t_{2} \rightarrow-\infty}} Z^{f}\left(t_{1}\right) S_{D}\left(t_{1}, t_{2}\right) Z\left(t_{2}\right)
$$

This procedure is an amplitude method in contrast to generalizations of the $\mathrm{BN}$ This procedure is an amplitude method in contrast to generalizations of the procedure, including the degenerate state method, 14,18 which are cross section methods. In QED and pertubative gravitation, the resulting $\mid \psi_{\mathrm{as}}>$ 's are essentially simple
generalizations $2,3,6,16,5$ of the well-known CS's, and form a space of coherent Fock states (CFS).

Explicit calculation in QCD showed ${ }^{6}$ that the IR divergences cancelled to $O\left(\alpha_{s}{ }^{2}\right)$ for quark scattering in a color-singlet $V_{\text {ext }}$. Later work showed ${ }^{16}$ that the above $O\left(\alpha_{s}{ }^{2}\right)$ for quark scattering in a color-singlet $V_{\text {ext }}$ Later work show soft IR finiteness to all orders in QCD by this CS method. Additional CS studies in QCD have been carried out. $24-26$

"Colorblind detectors and beams" are not necessary, as some have suggested, for this procedure gives meaning6,16 to color-specific reactions such as qq $\rightarrow \mathrm{qq}+$ gluon " averaging and there is a "coherent and colored, Fock state" asymptotically $\left|\Psi_{\mathrm{as}}\right\rangle=\exp [-\Omega]\left|\psi_{\mathrm{F}}\right\rangle$

In addition, a variation on the QCD parton model was proposed ${ }^{18}$ in which the partons are identified with nearly degenerate, coherent quark-gluon states. Schematically,

$$
\begin{aligned}
& |q\rangle \rightarrow|j\rangle=|q\rangle+|q g\rangle+|q g g\rangle \\
& +|q q \bar{q}>+l q q \bar{q} g\rangle+\ldots
\end{aligned}
$$

where "q" is a quark and " $\mathrm{g}$ " is a soft of hard collinear gluon or ghost. All kets in $\mid \mathrm{j}>$ have approximately the same four momentum. Explicit calculation was performed in deep-inelastic $" \mathrm{e}^{-}+$nucleon $\rightarrow \mathrm{e}^{-}+$anything" scattering (DIS) to next-to-leading-order including the $O(\alpha)$ finite parts. This verified the Kinoshita-Lee-Nauenberg ${ }^{14}$ theorem: "Partial transition probabilities computed by summing over nearly degenerate final states and initial states are free of all soft and collinear singularities."

To specify the initial state, a light-like "reference four-momentum" is introduced

$$
\mathrm{P}_{\text {ref }}^{\mu}(\mathrm{Q}) \text { with } \mathrm{P}^{2}=0, \overrightarrow{\mathrm{P}}=\overrightarrow{\mathrm{p}}+\sum_{\mathrm{i}} \overrightarrow{\mathrm{k}}_{\mathrm{i}}
$$

where $\vec{p}$ is for the initial quark, and the sum is over the initial IR gluons. With $q^{\mu}$ the virtual photon momentum, a generalized Bjorken $\mathrm{x}$ variable is defined as

$$
z \equiv-\frac{q^{2}}{2 P_{\text {ref }} \cdot q}=\frac{Q^{2}}{2 P_{\text {ref }} \cdot q}
$$

i.e. as the momentum fraction of a "jet", $\mid j>$, in the initial nucleon. The IR regions are:

$$
\begin{aligned}
& \text { SOFT : }|\overrightarrow{\mathrm{k}}| \leq \omega \ll \mathrm{Q}, \mu \\
& \text { HARD COLLINEAR : } \theta \leq \delta \ll 1, \omega<|\overrightarrow{\mathrm{k}}| \leq|\overrightarrow{\mathrm{p}}|
\end{aligned}
$$

Note that QCD has an intrinsic scale set by nature $\Lambda \approx 300 \mathrm{MeV}$ so in he context of bound state dynamics and constituent quarks $\delta$ and $\omega$ could be small, finite, and physical, e.g.

$$
\omega_{\text {soft }} \sim a \Lambda, \delta_{\text {collinear }} \sim b \frac{\Lambda}{Q}
$$

where $\mathbf{a}$ and $\mathbf{b}$ are dimensionless constants.

The calculation using the operator product expansion to $O\left(\alpha_{s}\right)$ was done for the nonsinglet structure functions $F_{2} \mathrm{NS}$ and for the associated operator matrix elements $\mathrm{A}_{\mathrm{p}}^{\mathrm{NS}}$. Diagramatically in $\mathrm{F}_{2} \mathrm{NS}\left(\mathrm{z}, \mathrm{Q}^{2}\right)$ besides the usual reals and virtuals, there are the care is necessary in evaluating the latter, e.g., the naive covariant Feynman rules do not provide the same terms that arise in the (correct) time-ordered Hamiltonian evaluation. The full $O\left(\alpha_{s}\right)$ terms in $F_{2}{ }^{N S}$ and in $A^{N S}$ do differ from the usual ones. Also, in each there are finite hard collinear gluon contributions going as $\ln (\delta / 2)$. However, the short-distance properties for DIS are the same as in the ordinary parton model because
the usual coefficient function $C_{n}^{N S}\left(Q^{2} / \mu^{2}, g^{2}\right)$ is obtained. Therefore, this CS formalism could provide a bridge between pertubative and nonpertubative treatments of QCD, e.g. in a lattice approach.

Coherence and Uncertainty Properties of the q-Analogue Quantized Radiation Field

The q-analogue CS's, $|z\rangle_{\mathrm{g}}$, can be used to investigate ${ }^{20}$ the properties of the $q$-analogue quantized radiation field in the $|z\rangle_{q}$ "classical limit" This parallels conventional quantum optics analyses. In the Heisenberg representation, we consider a specific mode of the generic $q$-analogue radiation field having a specific polarization $\hat{\epsilon}$, where ${ }^{19}$ for $0 \leq q \leq \infty$,

$$
a^{t}-q^{1 / 2} a^{t} a=q^{-N / 2}
$$

with $\left[N, a^{\dagger}\right]=a^{\dagger},[N, a]=-a$ and $[a, a]=0$. As $q \rightarrow 1$, these reduce to the usual boson commutation relations. We suppress both the subscript $\vec{k}$ and the polarization 
vectors $\hat{\epsilon}$ for the q-analogue electric and magnetic fields, etc.

The q-analogue CS satisfy

$$
a|z\rangle_{q}=z|z\rangle_{q}
$$

where $\mathrm{z}$ is a complex number. Up to a phase choice, they are

$$
\left.|z\rangle_{q}=N(z) \sum_{n=0}^{\infty} \frac{z^{n}}{\sqrt{ }[n] !} \ln \right\rangle_{q}
$$

where $N(z)=e_{q}\left(|z|^{2}\right)^{-1 / 2}$. In the $\ln >_{q}$ occupation number basis, $<m \ln >=\delta_{m n}$ and

$$
\mathrm{a}^{k} \ln >=\sqrt{[\mathrm{n}+1]} \ln +1>, \mathrm{a} \mid \mathrm{n}>=\sqrt{[\mathrm{n}]} \ln -1>
$$

with al0> $=0$. Note that the "bracket \#" is defined by

$$
\begin{aligned}
{[x]_{q} \equiv[x] } & =\frac{q^{x / 2}-q^{-x / 2}}{q^{1 / 2}-q^{-1 / 2}} \\
& =\frac{\sinh (s x / 2)}{\sinh (s / 2}
\end{aligned}
$$

and is called the "q-deformation" of $x(s=\log q)$. [x] is invariant under $q \leftrightarrow 1 / q$ so we can often consider $0<\mathrm{q}<1$. Notice that

$$
\begin{gathered}
N \ln >_{q}=n \ln >_{q}, a^{\dagger} a \ln >_{q}=[N] \ln >_{q}=[n] \ln >_{q}, \\
\operatorname{aa}^{\dagger} \ln >_{q}=[N+1] \ln >_{q}=[n+1] \ln >_{q} .
\end{gathered}
$$

The normalization and resolution of unity ${ }^{21,22}$ for these CS's involves the q-ixponential function

$$
e_{q}(z) \equiv \sum_{n=0}^{\infty} \frac{z^{n}}{[n] !}
$$

where $[n] !=[n] \mid n-1] \ldots[1],[0] !=1$. It is an entire function and $\left|e_{q y}(z)\right| \leq e_{q}(|z|) \leq$ $\exp (|z|)$. So this series representation converges uniformly and absolutcly for all tinite $z$ independent of the value of $q$. For $x>0, e_{q}(x)$ is positive, but for $x<0$ it is an alternating series. For $\mathrm{x}<0$ and $\mathrm{q}<\left(\mathrm{q}_{1}{ }^{*} \stackrel{\mathrm{q}}{\approx} 0.14\right)$ there is a universal behavior independent of the value of $q$ consisting of an infinite number of increasing amplitude oscillations of thecreasing frequency as $x \rightarrow(-\infty)$. For $q$ small and/or $n$ large, the asymptotic formula 27 for the zeros of $e_{q}(x)$ is

$$
\tilde{\mu}_{n}^{e}=-\frac{q^{1 / 2(1-n)}}{1-q} ; n=1,2, \ldots
$$

As $\mathrm{q}$ increases above the first collision point at $\mathrm{q}_{1}^{*} \approx 0.14$, these zeros collide in pairs and then move off the negative real axis into the complex $z$ plane.

These CS's $|z\rangle$ are good candidates for studying the classical limit of the generic q-analogue quantized radiation field because:

(i) They are "minimum uncertainty states" which minimize

$$
\mathrm{U}_{\mathrm{Q}, \mathrm{P}} \equiv \frac{2 \Delta \mathrm{Q} \Delta \mathrm{P}-\mathrm{K}[\mathrm{Q}, \mathrm{P}]>1}{\mathrm{~K}[\mathrm{Q}, \mathrm{P}>\mathrm{I}} \geq 0,
$$

as does the vacuum state $10>$. As usual

$$
\hat{\mathrm{Q}} \equiv(\hbar / 2 \omega)^{1 / 2}\left(\mathrm{a}^{\gamma}+\mathrm{a}\right), \hat{\mathrm{P}}=i(\hbar \omega / 2)^{1 / 2}\left(\mathrm{a}^{\dagger}-\mathrm{a}\right)
$$

(ii) The $\mathrm{n}^{\text {th }}$ order correlation function factorizes, i.e.

$$
\operatorname{Tr}\left(\rho E^{-}(x) E^{+}(y)=\mathscr{E}(x) \mathcal{E}^{+}(y), \ldots\right.
$$

(iii) There exists a resolution of unity $21,22,20$

$$
\int|z\rangle_{q}<z\left|d \mu(z)+\int \tilde{z}\right\rangle_{q}<\tilde{z} \mid d \tilde{\mu}=1
$$

and associated q-analogue diagonal operator representations analogous to the P-, Q-, and $\mathrm{W}$ - representations in quantum optics, e.g.

$$
\hat{\rho}=\int d \mu(z) \phi_{N}\left(z, z^{*}\right)\left|z>_{q} q^{<z}\right|
$$

$$
\left\langle\left(a^{t}\right)^{r} a^{s}\right\rangle=\operatorname{Tr}\left[\rho\left(a^{t}\right)^{r} a^{s}\right]=\int d \mu(z)\left(z^{*}\right)^{r} z^{r} \phi_{N}\left(z, z^{*}\right)
$$




$$
\left\langle\mathrm{a}^{\mathrm{r}}\left(\mathrm{a}^{\mathrm{t}}\right)^{\mathrm{s}}\right\rangle=\int \mathrm{d} \mu(\mathrm{z}) \mathrm{z}^{\mathrm{r}}\left(\mathrm{z}^{*}\right)^{\mathrm{s}} \psi_{\mathrm{N}}\left(\mathrm{z}, \mathrm{z}^{*}\right)
$$

$$
\psi_{N}\left(z, z^{*}\right) \equiv\langle z|\hat{\rho}| z\rangle=\int d \mu(y) \phi_{N}\left(y, y^{*}\right) N(y)^{2} N(z)^{2} e_{q}(y z)^{*} e_{q}\left(z y^{*}\right)
$$

In the completeness relation, for the measure

$$
d \mu(z)=1 / 2 \pi e_{q}\left(|z|^{2}\right) e_{q}\left(-|z|^{2}\right) d_{q}|z|^{2} d \theta
$$

the first term gives

$$
\int|z\rangle<z\left|d \mu=\sum_{n=0}^{\infty} \frac{1}{[n] !} \int_{0}^{\zeta_{i}} x^{n} e_{q}(-x) d_{q} x \ln ><n\right|
$$

The i-togral over $x=|z|^{2}$ is a $q$-integration over the interval from zero to some $\zeta_{i}>0$ ( $i$ fixed) where $-\zeta=-\zeta_{1}$ is the largest zero of $e_{q}(z),-\zeta_{2}$ the next, ... . The second term ${ }^{20}$ is due to a set of $q$-discrete auxiliary states $\mid \tilde{z}_{q}$ where $(k=0,1,2, \ldots)$

$$
\begin{aligned}
& \tilde{a}_{k}\left|\tilde{z}_{k}\right\rangle=\left(q^{1 / 4} \tilde{z}_{k}\right)\left|\tilde{z}_{k}\right\rangle \\
& \left.\left|\tilde{z}_{k}\right\rangle=M_{k} \sum_{j=0}^{\infty} \frac{\left(q^{1 / 4} \tilde{z}_{k}\right)^{j}}{\sqrt{ }[j] !} j+k\right\rangle
\end{aligned}
$$

where $M_{k}=e_{q}\left(q^{1 / 2} x_{k}\right)^{-1 / 2},\left|\tilde{z}_{k}\right|^{2} \equiv x_{k}=q^{k / 2} \zeta_{i}$. For each $k, \tilde{a}_{k}$ and $\tilde{a}_{k}^{k}$ are defined on an infinite dimensional subspace $|k+j\rangle, j \geq 0$, and satisfy a quantum algebra

$$
\tilde{a}_{k} \tilde{a}_{k}^{x}-q^{1 / 2} \tilde{a}_{k}^{x} \tilde{a}_{k}=q^{-\tilde{N}_{k} / 2}
$$

in this subspace. With the discrete measure

$$
d \tilde{\mu}_{k}=\frac{1}{2 \pi M_{k}^{2}} e_{q}\left(-\left.\tilde{z}_{k}\right|^{2}\right) d \theta
$$

the second term in the completeness relation gives

$$
\int|\tilde{z}\rangle\langle\tilde{z}| d \tilde{\mu}=\sum_{n=0}^{\infty} \sum_{k=0}^{n} \frac{1}{[n-k] !}\left(q^{1 / 2} x_{k}\right)^{n-k} \ln >\langle n| e_{q}\left(-x_{k}\right)
$$

The resolution of unity is satisfied since

$$
\int_{0}^{\zeta_{i}} e_{q}(-x) x^{n} d_{q} x=[n] !\left\{1-\sum_{k=0}^{n} \frac{\left(q^{1 / 2} x_{k}\right)^{n-k}}{[n-k] !} e_{q}\left(-x_{k}\right)\right\}
$$

is the q-analogue of Euler's formula 21,20 for $\Gamma(x)$.

Using these q-analogue CS's, we next study, 20,21 the classical limit for various quantities characteristics of "q-boson statistics"? Because $[a, a]=0$, for the number Hamiltorian $H_{n}=\hbar \omega(N+1 / 2)$ the usual Planck distribution still follows ${ }^{28}$ for a free q-boson gas. However, for $q \neq 1$ the $|z\rangle_{q}$ do not give a Poisson number distribution, corresponding to statistically independent sources, since

$$
\begin{aligned}
P_{n}(z) & \equiv \mid \underset{q}{|<n| z>\left._{q}\right|^{2}} \\
& =\frac{|z|^{2 n}}{[n] ! e_{q}\left(|z|^{2}\right)}
\end{aligned}
$$

so for fixed $|z|$, as $q$ decreases the peak narrows and shifts to smaller $n$. Table 1 gives the associated number operator and phase operator uncertainties. We will discuss the phase operator $\phi_{\mathrm{q}}$ below. [Numerically the constant $\eta_{.7} \approx 2.4$ and $\eta_{.06} \approx 0.9$ \})

Note that the fractional uncertainty for $\mathrm{N}$ still decreases as $|z|$ increases because the mean value $\langle N\rangle=\langle z|N| z\rangle$ still increases, although with a smaller slope as $q$ decreases:

$$
\begin{aligned}
\langle N\rangle & \left.=2 / s<\sinh ^{-1}\{[N] \sinh (s / 2)\}\right\rangle \\
& \rightarrow 2 \alpha_{q} \log |z|+\beta_{q}, \quad|z|^{2} \text { large }
\end{aligned}
$$

The last line is a crude numerical estimate with $\alpha_{q}, \beta_{q} q$-dependent constants. Also, the important ratio of "the variance to the mean value" is distinctly different from that for known photon statistics, see Table 2: 
Table 1: $\quad \mathrm{N}$ and $\hat{\phi}$ uncertainties in $|z\rangle_{\mathrm{q}}$ classical limit

\begin{tabular}{lcc}
\hline Uncertainty & Behavior as $|z| \rightarrow \infty$ when $q \neq 1$ & Case $q=1$ \\
\hline$\Delta N$ & $\eta_{q}=\left\{\begin{array}{l}q \text {-dependent } \\
\text { constant }\end{array}\right.$ & $|z|$ \\
$\hat{\Delta} \phi_{q}$ & $\frac{1}{2 \eta_{q}}$ & $\frac{1}{2|z|}$ \\
$\Delta N \Delta \hat{\phi}_{q}$ & $\frac{1}{2}$ & $\frac{1}{2}$ \\
$\frac{\Delta N}{\langle N\rangle}$ & $\frac{2 \eta_{q}}{2 \alpha_{q} \log |z|+\beta_{q}}$ & $\frac{1}{|z|}$ \\
$\frac{\Delta \phi_{q}}{k \phi_{q}>1}$ & $\frac{1}{2 \eta_{q} k \phi_{q}>}$ & $\frac{1}{2|z| k \phi>\mid}$ \\
\hline
\end{tabular}

Table 2: Comparison with known photon statistics.

\begin{tabular}{lll}
\hline Bose-Einstein & $\frac{(\Delta N)^{2}}{\langle N\rangle}=\langle N+1\rangle$ & Thermal Source \\
Poisson statistics & $\frac{(\Delta N)^{2}}{\langle N\rangle_{|z|} \rightarrow \infty} \begin{array}{ll}\text { Laser Light } \\
(\mathrm{q}=1 \text { CS's })\end{array}$ \\
q-Boson statistics & $\frac{(\Delta N)^{2}}{\langle N\rangle_{|z|} \rightarrow \infty}$ & $q \neq 1$ CS's \\
\hline
\end{tabular}

Other fractional uncertainties, $\Delta \hat{0} \mid\langle\hat{0}\rangle$, in the $|z\rangle_{q}$ classical limit can be studied analytically because the "resolution function" $\lambda(z)$ is related to the q-exponential function

$$
\begin{aligned}
\lambda(z) & =\langle z|\Lambda| z\rangle \equiv\langle z|[N+1]| z\rangle-\langle z|[N]| z\rangle \\
& =\left(q^{-1 / 2}-1\right)|z|^{2}+e_{q}\left(q^{1 / 2}|z|^{2}\right) / e_{q s}\left(|z|^{2}\right)
\end{aligned}
$$

Thus, $\lambda(z)$ diverges linearly

$$
\lim _{z z \rightarrow \infty} \lambda(z) \rightarrow\left(q^{-1 / 2}-1\right)|z|^{2}+\epsilon_{1}(q)
$$

where the non-negative q-dependent constants

$$
\epsilon_{1} \equiv \lim _{|z| \rightarrow \infty}\left\{\left(e_{q}\left(\left.q\right|^{1 / 2}|z|^{2}\right) / e_{q}\left(|z|^{2}\right)\right\}, \epsilon_{2} \equiv \lim _{|z| \rightarrow \infty}\left\{e_{q}\left(q|z|^{2}\right) / e_{q}\left(|z|^{2}\right)\right\} .\right.
$$

Note $\epsilon_{i}<1$ for $q \neq 1$, and $\epsilon_{i} \rightarrow 1$ as $q \rightarrow 1$. For arbitrary $|z|, \lambda(z)$ is bounded

$$
\left\{1,\left(q^{-1 / 2}-1\right)|z|^{2}\right\} \leq \lambda(z) \leq\left\{\left(q^{1 / 2}-1\right)|z|^{2}+1\right\} .
$$

Note that this resolution function characterizes both the $\hat{Q}$ and $\hat{P}$ uncertainty relation

$$
\mathrm{q}\langle\mathrm{z}|[\hat{\mathrm{Q}}, \hat{\mathrm{P}}]| \mathrm{z}\rangle_{\mathrm{q}}=i \hbar \lambda(\mathrm{z}) \geq i \hbar
$$

and also the variances of the generic $\vec{E}$ and $\vec{B}$ fields

$$
\left.(\Delta \hat{\mathrm{E}})^{2}\right|_{b D}=\left.(\Delta \hat{\mathrm{B}})^{2}\right|_{D D}=\frac{\hbar \omega}{2 \epsilon_{0} V} \lambda(\mathrm{z}) \geq \frac{\hbar \omega}{2 \epsilon_{0} V}
$$

The top part of Table 3 shows that the fractional uncertainties of these quantities, which characterize the q-analogue radiation field, are all of order one, and only vanish as $1 /|z|$ when $q=1$.

The last line of Table 3 shows that the fractional uncertainty is still zero for both the usual $\mathrm{N}$ operator and for the $\mathrm{N}$-Hamiltonian 


$$
\begin{aligned}
H_{N} & \equiv \hbar \omega(N+1 / 2) \\
\left\langle H_{N}>\right. & =\hbar \omega\langle N+1 / 2> \\
& \rightarrow 2 \hbar \omega \alpha_{q} \log |z|+\ldots,|z|^{2} \text { large }
\end{aligned}
$$

\begin{tabular}{|c|c|c|c|}
\hline Quantity & $\hat{0}$ & $\frac{\Delta \hat{0}}{k \hat{0}>1}$ as $|z| \rightarrow \infty$ & Case $q=1$ \\
\hline Position & $\hat{Q}$ & $\frac{1}{2 \cos \theta} \sqrt{\left(q^{-1 / 2}-1\right)+\epsilon_{1} /|z|^{2}}$ & $\frac{1}{2 \cos \theta|z|}$ \\
\hline Momentum & $\hat{\mathrm{P}}$ & $\frac{1}{2 \sin \theta} \sqrt{\left(q^{-1 / 2}-1\right)+\epsilon_{1} /|z|^{2}}$ & $\frac{1}{2 \sin \theta|z|}$ \\
\hline$\vec{E}, \vec{B}$ Fields & $\hat{\mathrm{E}}, \hat{\mathrm{B}}$ & $\frac{1}{2} \sqrt{\left(q^{-1 / 2}-1\right)+\epsilon_{1} /|z|^{2}}$ & $\frac{1}{2|z|}$ \\
\hline Bracket \# & {$[\mathrm{N}]$} & $\sqrt{\left(q^{-1 / 2}-1\right)+\epsilon_{1} /|z|^{2}}$ & $\frac{1}{|z|}$ \\
\hline Bracket (\#+1) & {$[N+1]$} & $\sqrt{\left(q^{-1 / 2}-1\right)+\epsilon_{1} /|z|^{2}(-1+2 q)+\ldots}$ & $\frac{1}{|z|+1 / z \mid}$ \\
\hline $\left.\begin{array}{l}\text { Quadratic } \hat{\mathrm{P}}, \hat{\mathrm{Q}} \\
\text { Hamiltonian }\end{array}\right\}$ & $\mathrm{H}_{\mathrm{P}, \mathrm{Q}}$ & $\sqrt{\left(q^{-1 / 2}-1\right)+\epsilon_{1} /|z|^{2}\left(-1+2 q^{1 / 2} \ldots\right.}$ & $\frac{1}{|z|+1 / z \mid}$ \\
\hline $\begin{array}{l}\text { \# Operator and } \\
\mathrm{N} \text {-Hamiltonian }\end{array}$ & $\left.\begin{array}{l}\mathrm{N} \\
\mathrm{H}_{\mathrm{N}}\end{array}\right\}$ & zero & $\frac{1}{|z|}$ \\
\hline
\end{tabular}

by Eq. (35b). Unlike for the single-mode's quadratic $\hat{\mathrm{P}}, \hat{\mathrm{Q}}$ Hamiltonian

Table 3: Fractional uncertainties for the q-analogue quantized radiation field.

$$
H_{P, Q}=1 / 2 \hbar \omega\left(a^{\dagger} a+a a^{\dagger}\right)=1 / 2\left(\hat{P}^{2}+\omega^{2} \hat{Q}^{2}\right),
$$

$\mathrm{H}_{\mathrm{N}}$ does have the orthodox free-field limit, the usual free-quanta additivity in the energy and the usual mathematical independence from the basic commutation relation [a, $a$ when $q \neq 1$. All of these properties are absent for $H_{p}$ of Eq. (43). However, the deviation of the resolution function $\lambda(z)$ from " 1 " does depend on the deviation of $E_{\bar{a}} \equiv\left\langle z\left|H_{P, Q}\right| z\right.$ which involves Eq. (43), from the vacuum, or ground state, energy $\hbar \omega / 2$ where $\overline{\mathbf{n}}=\langle z|N| z\rangle$ or $\langle z|[N]| z\rangle$.

Mathematically a Hermitian phase operator conjugate to $N$, or to $[N]=a^{t_{a}}$ does not exist. Nevertheless, as in the literature in quantum optics, it is very useful to study the different phase definitions ${ }^{29}$ for the $\mathrm{q}$-analogue quantum field and the approximate number-phase commutation relation. ${ }^{30}$ An exp $(i \phi)_{q}$ generalization of the Susskind-Glogower phase operator ${ }^{29}$ is defined by

$$
\mathrm{a} \equiv([\mathrm{N}+1])^{1 / 2} \exp (i \phi), \mathrm{a}^{\nmid} \equiv \exp (-i \phi)([\mathrm{N}+1])^{1 / 2} \text {. }
$$

So. there are the Hermitian operators

$$
\begin{aligned}
\operatorname{côs}(\phi) & \equiv 1 / 2\{\exp (i \phi)+\exp (-i \phi)\} \\
\sin (\phi) & \equiv(1 / 2 i)\{\exp (i \phi)-\exp (-i \phi)\}
\end{aligned}
$$

In the $\ln >_{q}$ basis, these deĩnitions correspond to

$$
\exp (i \phi)_{\mathbf{q}} \equiv \sum_{n=0}^{\infty}|n><n+1|
$$

which is manifestly q-independent in this basis, non-unitary, and obviously the which is manifestly q-independent operator ${ }^{29}$ is obtained by introducing a complete, orthonormal basis of $(s+1)$ phase states

$$
\left.\theta_{\mathrm{m}}>=(s+1)^{-1 / 2} \sum_{\mathrm{n}=0}^{\mathrm{S}} \exp \left(i \mathrm{n} \theta_{\mathrm{m}}\right) \ln \right\rangle_{\mathrm{q}}
$$

with $\theta_{m}=\theta_{0}+2 m \pi /(s+1)$ with $m=0,1, \ldots, s$ where $\theta_{0}$ a reference, indicial phase. These are eigenkets of the Hermitian

$$
\hat{\phi}_{\mathrm{q}} \equiv \sum_{\mathrm{m}=0}^{\mathrm{s}} \theta_{\mathrm{m}}\left|\theta_{\mathrm{m}}><\theta_{\mathrm{m}}\right|
$$

and of the unitary 
$\exp (i \phi)_{q} \equiv|0\rangle<1|+| 1><2|+\ldots+| s-1><s\left|+\exp \left(i(s+1) \theta_{0}\right)\right| s><0 \mid$

The latter only differs from SG by the last term. These operators are also manifestly $q$-independent in the $\ln \rangle_{q}$ basis so the non-trivial $q$-analogue phase effects in the $|z\rangle_{q}$ classical limit arise due to the $q$-dependence of the mapping $\ln \rangle_{q} \Rightarrow \mid \nabla_{q}$. $|z| \mathrm{e}^{i \theta}$

Various, standard, ${ }^{29}$ number-phase ul:certainty relations hold for arbitrary q: $(z=$

$$
\mathrm{Q}\left(|z|^{2}, \theta\right) \equiv \frac{(\Delta N)^{2}(\Delta \sin (\phi))^{2}}{<\cos (\phi)\rangle^{2}} \geq \frac{1}{4}
$$

and the associated Carruthers-Nieto relation

$$
U\left(|z|^{2}\right)=(\Delta N)^{2} \frac{\left\{(\Delta \sin (\phi))^{2}+(\Delta \cos (\phi))^{2}\right.}{\left\{\langle\sin (\phi)\rangle^{2}+\langle\cos (\phi)\rangle^{2}\right\}} \geq \frac{1}{4} .
$$

But, for $|z|$ large although both $Q$ and $U$ approach constants, the $|z\rangle_{q}$ do not minimize these relations except for $q=1$. Similarly, $\sin (\phi)$ and côs $(\phi)$ do not commute for $|z|$ large (i.e. $\Delta \sin (\phi) \Delta$ côs $(\phi)$ doesn't vanish except for $q=1$ ), in spite of some correspondence behavior

$$
\begin{gathered}
\frac{\langle z|\sin (\phi)| z\rangle}{\langle z|\operatorname{côs}(\phi)| z\rangle}=\frac{\sin (\theta)}{\cos (\theta)} \\
\left\langle z\left|\cos (\phi)^{2}+\sin (\phi)^{2}\right| z\right\rangle=1-1 / 2 e_{q}\left(|z|^{2}\right)^{-1} \rightarrow 1 \text { for }|z| \rightarrow \infty
\end{gathered}
$$

Most remarkably the conventional number-phase uncertainty relation ${ }^{30}$ of Dirac does still follow for the generic q-analogue quantum field

$$
\Delta \mathrm{N} \Delta \hat{\phi}_{\mathrm{q}}=1 / 2
$$

in the $|z|_{q}$ classical limit. Here, and in Table $1, \phi_{q}$ is the q-generalization of the PB phase operator. The properties of $\phi_{\mathrm{q}}$ in the $|\mathrm{z}\rangle_{\mathrm{q}}$ basis depend on the $\theta_{\mathrm{m}}$-angular probability function

$$
\left.\bar{P}_{q}\left(\theta_{m}\right) \equiv \lim _{s=\infty}(s+1)\left|<\theta_{m}\right| z\right\rangle_{q} R
$$

which is the phase analogue of $P_{n}(|z|)$ considered above. The mean is $q$-independent

$$
\left\langle\hat{\phi}_{q}\right\rangle \equiv 1 / 2 \pi \int_{0}^{2 \pi} \theta_{m} \bar{P}_{q}\left(\theta_{m}\right) d \theta_{m}=\theta
$$

where $z=|z| \exp (i \theta)$. But the variance for $|z|$ large only falls as $(1 / 2|z|)^{2}$ when $q=1$; for $q \neq 1$ it drops to the constant value $\left(1 / 2 \pi_{q}\right)^{2}$. In general,

$$
\left(\Delta \hat{\phi}_{q}\right)^{2}=\pi^{2} / 3+4(N(z))^{2} \sum_{n>l}^{s} \frac{(-)^{n+l}}{(n-l)^{2}}\left\{\frac{|z|^{n+l}}{\sqrt{ }[n] ![l !}\right\}
$$

Note that $\bar{P}_{q}\left(\theta_{m}\right)$ is normalized by

$$
1 / 2 \pi \int_{0}^{2 \pi} \overline{\mathrm{P}}\left(\theta_{\mathrm{m}}\right) \mathrm{d} \theta_{\mathrm{m}}=1
$$

and that for $|z|$ large for $q=1$

$$
\lim _{|z| \rightarrow \infty} \bar{P}\left(\theta_{m} \rightarrow 2 \pi \delta\left(\theta-\theta_{m}\right) \quad(q=1)\right.
$$

which involves the Dirac delta distribution. However, for $q \neq 1$, instead

$$
\lim _{|z| \rightarrow \infty} \vec{P}(\theta) \rightarrow 2 \pi \delta_{q}\left(\theta-\theta_{m}\right) \quad(q \neq 1)
$$

where $\delta_{q}\left(\theta-\theta_{m}\right)$ is a "bell-shaped" function with finite width $\left(\Delta \phi_{q} \rightarrow 1 / 2 \eta_{q}\right)$ and finite height.

In the |\rangle$_{q}$ basis, this $\bar{P}_{q}\left(\theta_{m}\right.$ function also appears ${ }^{29}$ in Dirac's approximate number-phase commutation relation

$$
\mathrm{q}_{\mathrm{q}}\left\langle\mathrm{z}\left|\left[\mathrm{N}, \hat{\phi}_{\mathrm{q}}\right]\right| z\right\rangle_{\mathrm{q}}=i-i \overline{\mathrm{P}}_{\mathrm{q}}(\theta)
$$

So for large $|z|$, for $q \neq 1$

$$
\lim _{|z| \rightarrow \infty} \mathrm{q}^{<z \mid}\left[\mathrm{N}, \hat{\phi}_{\mathrm{q}}\right]|\mathrm{z}\rangle_{\mathrm{q}}=i-i 2 \pi \delta_{\mathrm{q}}\left(\theta-\theta_{\mathrm{o}}\right) \quad(\mathrm{q} \neq 1)
$$


for $\phi_{\mathrm{q}}$ eigenvalues from the indicial $\theta_{0}$ to $\left(\theta_{0}+2 \pi\right)$. This extra-term which depends on $\delta_{1}\left(\theta-\theta_{0}\right)$ resembles a smeared "magnetic monopole" string in that it serves to uniquely specify the phase angle. For $q=1$, the smearing is absent.

Thus, (i) the q-boson CS's $|z\rangle_{q}$ both give and minimize $\left(\Delta N \Delta \phi_{q}=1 / 2\right)$ the approximate $\left[\mathrm{N} \phi_{\mathrm{q}}\right] \approx i$, and (ii) for the $\mathrm{q}$-boson quantized radiation field, in contrast to the standard $\hat{\mathrm{Q}}$ and $\hat{\mathrm{P}}$ operators, the operators $\mathrm{N}$ and $\hat{\phi}_{\mathrm{q}}$ turn out to be almost canonically conjugate in the $|z\rangle_{\mathrm{q}}$ classical limit.

\section{Projector Definition of the Usual Coherent States and Squeezed States}

From the perspective of future applications of CS's, it is important to have several definitions, though equivalent, of the usual CS's for sometimes one definition will generalize whereas another will not. For example, it's the eigenstate of "a" definition which simply generalizes in the case of q-bosons where the explicit analogue of the $D(z)=\exp \left(z^{2}-z a\right)$ operator is unknown.

The projector idea ${ }^{23,} 31$ is very simple. Recall in the case of the CS's that the Baker-Campbell-Hausdorff formula gives

$$
\mathrm{aD}(\mathrm{z})=\mathrm{D}(\mathrm{z})(\mathrm{a}+\mathrm{z})
$$

So, for the bose operators $b=a-z, b^{k}=a^{k}-z^{*}$,

$$
\mathrm{bD}(\mathrm{z})=\mathrm{D}(\mathrm{z}) \mathrm{a} \text {, }
$$

Thus, the CS $|z\rangle$ is a vacuum state $10>_{b}$ of the "b bosons"

$$
b|z\rangle=b D(z)|0\rangle=0 \Rightarrow|z\rangle=10\rangle_{b} \text {. }
$$

This means $D(z)$ projects the "old vacuum" state $10>=10>_{a}$ into the "new vacuum" state $10>_{b}$. But, other operators can be used as such a "projector", for instance ${ }^{31}$

$$
P=\Sigma \frac{(-1)^{n} b^{X_{n}} b^{n}}{n !}
$$

where $b P=0, P^{2}=P$. For $b \equiv a-z, P \mid 0>2$ is the usual $C S$ up to a normalization constant. For the usual squeezed vacuum states, the same $P$ can be used except now $b \equiv \mu a+v a^{t}, b^{t}=\mu^{*} a^{t}+v^{*} a$, so

$$
\begin{aligned}
P|0\rangle_{a} & =\left(1-1 / 2|v|^{2}+3 / 8|v|^{4}-5 / 16|v|^{6}+\ldots\right) \mid 0>_{a} \\
& -\left(1 / 2-3 / 4|v|^{2}+5 / 16|v|^{4}-35 / 32|v|^{6}+\ldots\right) \mu^{*} v a^{t 2} \mid 0>_{a} \\
& +\cdots \\
& =\left(1-|\zeta|^{2}\right)^{1 / 2} \exp \left(-\zeta a^{t} a^{t} / 2\right) \mid 0>_{a}
\end{aligned}
$$

For the new $b$ bosons the parameters $\mu=\cosh |\alpha|, v=e^{i \phi}$ sinh $|\alpha|$ with $\zeta=e^{i \phi}$ tgh $|\alpha|$ and $\alpha=\mid \alpha e^{i \phi}$. Similarly, the usual "coherent squeezed state" is defined by use of two projectors

$$
\begin{aligned}
|z, \alpha\rangle & =|\beta ; \mu, v\rangle \\
& =e^{|\beta|^{2} / 2}\left(1-|\zeta|^{2}\right)^{-1 / 4}{\left.\underset{c=b-\beta}{P}\right|_{b=\mu a+v a t}|0\rangle_{2}}^{P}
\end{aligned}
$$

where $\beta=\mu z+v z^{*}$.

Since this procedure gives the $b$ bose vacuum state $10>_{b}$, the Fock state for an arbitrary number " $n$ " of $b$ bosons is also known

$$
\ln >_{b}=\frac{b^{t_{n}}}{\sqrt{n !}} 10>_{b}
$$

If $|0\rangle_{b}$ corresponds to the $C S$, then $\left.\ln \right\rangle_{b}$ is called a coherent Fock state (CFS). If 10$\rangle_{b}$ corresponds to the squeezed vacuum state, then $\ln \rangle_{b}$ is a squeezed Fock state (SFS).

In the case of a q-bosons, vacuum projectors are known explicitly and are simple for both realizations of the q-boson commutation relations. ${ }^{32}$

\section{Acknowledgment:}

This work was supported by the NYS/UUP PDQWL Faculty Award Program, by U.S. Department of Energy Grant No. DE-FG02-86ER40291, and by the High Energy Physics Theory Group at Argonne National Laboratory. 
References

1. F. Bloch and A. Nordsieck, Phys. Rev. 52 (1937) 54; D.R. Yennie, S.C Frautschi, and H. Suura, Ann. Phys. (N.Y.) 13 (1961) 379; J. Schwinger, Phys. Rev 76 (1949) 290 S. Weinberg R.R Yennie Com. $56(1990) 351$.

2. J. Do (1. Thys. 5 (1964) 729. V Chung Phys. Rev 140 (1965) B1110 M. Greco and G. Rossi, Nuovo Cim. 50 (1967) 168; T. Kibble, J. Math. Phys. 9 M. Greco and G. Rossi, Nuovo Cim. $\frac{50}{1527}$ (1967) 168; T. Kibble, J. Math. Phys. (1968) 315; Phys. Rev. 173 (1968) 1527; $\frac{175}{\text { N. Papanicolaou, Phys. Rep. } 24 \text { (1976) }}$ D7 (1973) 1082; D11 (1975) 3481, 4504; N. Papanicolaou, Phys. Rep. 24 (1976)

4 (1971) 745]. Ther. Phys. 29 (1960) 1109; J. Storrow, Nuovo Cim. 54A T. Morota, Prog. Theor. Phys. 29 (1960) 1109; J. Storrow, Nuovo Cim 54A (1968) 15; Ph. Blanchard, Comm. Math. Phy. 15 (19) "Theory of Photons and Phys. Rev. D7 (19730 3114; J.M. Jauch and

Electons," 2nd ed. (Springer, N.Y. 1976).

5. H.F. Contopanagos and M.B. Einhom, Phys. Rev. D45 1978) 1196; C.A. Nelson, Nucl. D.R. Butler and C.A. Nelson, Phys. Rev. D18 (1978) 1196; C.A. Nelson, Nucl. Phys. B181 (1981) 141

7. J. Frenkel, J.G.M. Gatheral, and J.C. Taylor, Nucl. Phys. B194 (1982) 172; C.A. Nelson, Nucl. Phys. B221 (1983) 431.

8. G. Sterman and S. Weinberg, Phys. Rev. Lett. 39 (19770 1436

9. R.P. Feynman, "Photon-Hadron Interactions," (Benjamin, Reading, MA, 1972).

10. D. Amati, R. Petronzio, and G. Veneziano, Nucl. Phys. B140 (1978) 54; R.K Ellis, H. Georgi, M. Machacek, H.D. Politzer, and G.G. Ross, ibid. B152 (1979) 285; S. Libby and G. Sterman, Phys. Rev. D18 (1978) 3705; J.C. Collins, D.E. Soper and G. Sterman, Phys. Lett. 134B (1984) 263; G.T. Bodwin, Phys. Rev. D31 (1985) 2616; S. Catani, M. Ciafaloni, and F. Hauptmann, Phys. Lett. B307 (1993) 147 .

11. A H. Mueller "Perturbative OCD" (World Sci., Singapore, 1989); T. Muta, "Foundations of QCD," (World Sci., Singapore, 1987).

12. R. Doria, J. Frenkel and J.C. Taylor, Nucl. Phys. B168 (1980) 93.

13. C. Di'Lieto, S. Gendron, I.G. Halliday, and C.T. Sachrajda, Nucl. Phys. B183 (1981) 223; A. Andrasi, M. Day, R. Doria, J. Frenkel, and J.C. Taylor, ibid B182 (1981) 104 .

14. T. Kinoshita, J. Math. Phys. 3 (1962) 650; T.D. Lee and M. Nauenberg, Phys Rev. 133 (1964) B1549; and see N. Nakanishi, Prog. Theo. Phys. 19 (1958) 159.

I. Prog Theor. Phys 65 (1981) 1466; N. Yoshida, ibid. 66 (1981) 269.

C.A. Nelson, Nucl. Phys. B186 (1981) 187.

C.A. Nelson, Phys. Lett. $177 \mathrm{~B}$ (1986) 93. A. Axelrod and C.A. Nelson, Phys. Rev. D32 (1985) 2385; T. Muta and C.A. Nelson, ibid. D25 (1982) 2222.

19. A. MacFarlane, J. Phys. A22 (1989) 4581; L. Biedenharn, ibid A22 (1989) L873 C.-P. Sun and H.-C. Fu, ibid A22 (1989) L983; M. Chaichian and P. Kulish, Phys. Lett. B234 (1990) 72; and C. Zachos, Cont. Math. 134 (1992) 351.

20. C.A. Nelson, SUNY BING 9 $7 / 92$ (to appear in "Symmetries in Science VI," ed. B. Gruber); and C.A. Nelson and M.H. Fields, SUNY BING 8/1/93.

21. R.W. Gray and C A. Nelson, J. Phys A23 (1990) L945; S.-H. Chiu, R W. Gray, and C.A. Nelson, Phys. Lett. A164 (1992) 237; A.J. Bracken, D.S. McAnally,
R.B. Zhang, and M.D. Gould, J. Phys. A24 (1991) 1379; B. Jurco, Lett. Math. Phys. 21 (1991) 51

23. Z. Yu and C.A. Nelson, SUNY BING 6/1/93.

24. M. Ciafaloni, Phys. Lett. 150B (1985) 379; S. Catani, M. Ciafaloni, and G. Marchesini, Nucl. Phys. B264 (1986) 586.

25. V. del Duca, L. Magnea and G. Sterman, Nucl. Phys. B324 (1989) 391; H.F. Contopanagos, ibid. B397 (1993) 539.

26. M. Greco, F. Palumbo, G. Pancheri-Srivastava and Y. Srivastava, Phys. Lett. 77B (1978) 282; H.D. Dahmen and F. Steiner, Z. Phys. Ci1 (1981) 247.

27. CA Nelson and M.G. Gartley, SUNY BING 6/16/93.

28 R Bullough and N. Bogoliubov, J. Phys. A25 (19920 4057; G. Agarwal and S. R. Bullough and N. Bogolieov, A7 (1992) 2407; S. Vokos and C. Zachos, Chaturvedi, Mod. Phys. Lett. $\frac{A 7}{M}$. Chaichian, R. Gonzalez Felipe, and C. ANL-HEP-CP-93-39 (1993); $M$

29. L. Susskind and J. Glogower, Phys. 1 (1964) 49; W.H. Louisell, Phys. Lett. (1963) 60; P. Carruthers and M.M. Nieto, Phys. Rev. Lett. 14 (1965) 385; Rev. Mod. Phys. 40 91968) 411; D.T. Pegg and S.M. Barnett, Europhys. Lett. (1988) 483; R. Lynch. Phys. Rev. A41 (1990) 2841; M. Chaichian and D. Ellinas, J. Phys. A23 (1990) L291; J.W. Noh, A. Fongeres, and L. Mandel, Phys. Rev. Lett. 67 (1991) 1426)

30. P. Heitler, "Quantum .A. Dirac, Proc. Roy. Soc. London 1954); P.W Anderson "Basic Notions of Theory of Rater Physics" (Addison-Wesley, 1984)

31. Theo. Phys. 31 (1964) T. Marumori, M. Yam; 37 (1967) 336.

32. Z. Yu, Phys. Lett. A175 (1993) 391; Fan Hong-Yi and Jing Ci-cong, China Uni. of Sci. \& Tech. Preprint, Hefei, China (1993)

$$
\text { Prypents/tinnowd }
$$

\section{DISCLAIMER}

This This report was prepared as an accour their Government. Neither the United States Government nor any agency the liability or responsiemployees, makes any warranty, express or implied, or assumes any legal liability or respet bility for the accuracy, completeness, or usefuless of any information, apparatus, pro privately owned rights. Referprocess disclosed, or represe trade name, trademark ence herein to any specific conmercial prester im manufacturer, or otherwise does not necessarily constive mendation, or favoring by the United States Government or any agency the the of and opinions of authors expressed herein do not United States Government or any agency thereof. 


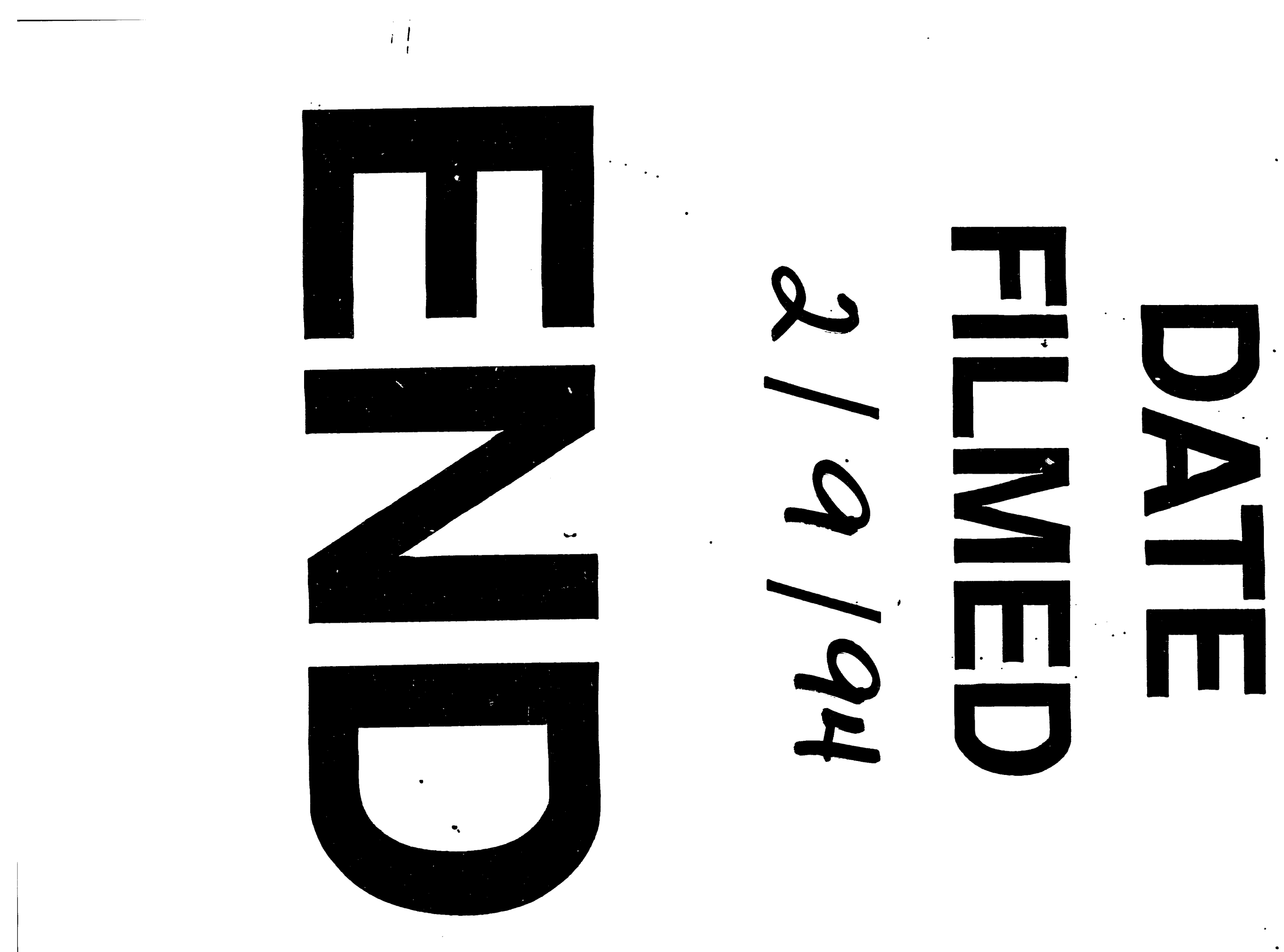

